\title{
On two problems in graph Ramsey theory
}

\author{
David Conlon* Jacob Fox ${ }^{\dagger} \quad$ Benny Sudakov ${ }^{\ddagger}$
}

\begin{abstract}
We study two classical problems in graph Ramsey theory, that of determining the Ramsey number of bounded-degree graphs and that of estimating the induced Ramsey number for a graph with a given number of vertices.

The Ramsey number $r(H)$ of a graph $H$ is the least positive integer $N$ such that every twocoloring of the edges of the complete graph $K_{N}$ contains a monochromatic copy of $H$. A famous result of Chvátal, Rödl, Szemerédi and Trotter states that there exists a constant $c(\Delta)$ such that $r(H) \leq c(\Delta) n$ for every graph $H$ with $n$ vertices and maximum degree $\Delta$. The important open question is to determine the constant $c(\Delta)$. The best results, both due to Graham, Rödl and Ruciński, state that there are constants $c$ and $c^{\prime}$ such that $2^{c^{\prime} \Delta} \leq c(\Delta) \leq 2^{c \Delta \log ^{2} \Delta}$. We improve this upper bound, showing that there is a constant $c$ for which $c(\Delta) \leq 2^{c \Delta} \log \Delta$.

The induced Ramsey number $r_{i n d}(H)$ of a graph $H$ is the least positive integer $N$ for which there exists a graph $G$ on $N$ vertices such that every two-coloring of the edges of $G$ contains an induced monochromatic copy of $H$. Erdős conjectured the existence of a constant $c$ such that, for any graph $H$ on $n$ vertices, $r_{i n d}(H) \leq 2^{c n}$. We move a step closer to proving this conjecture, showing that $r_{i n d}(H) \leq 2^{c n \log n}$. This improves upon an earlier result of Kohayakawa, Prömel and Rödl by a factor of $\log n$ in the exponent.
\end{abstract}

\section{Introduction}

Given a graph $H$, the Ramsey number $r(H)$ is defined to be the smallest natural number $N$ such that, in any two-coloring of the edges of $K_{N}$, there exists a monochromatic copy of $H$. That these numbers exist was first proven by Ramsey [30] and rediscovered independently by Erdős and Szekeres [17. Since their time, and particularly since the 1970s, Ramsey theory has grown into one of the most active areas of research within combinatorics, overlapping variously with graph theory, number theory, geometry and logic.

The most famous question in the field is that of estimating the Ramsey number $r(t)$ of the complete graph $K_{t}$ on $t$ vertices. However, despite some small improvements [32, 5], the standard estimates, that $2^{t / 2} \leq r(t) \leq 2^{2 t}$, have remained largely unchanged for over sixty years. Unsurprisingly then, the field has stretched in different directions. One such direction that has become fundamental in its own right is that of looking at what happens to the Ramsey number when we are dealing with various types of sparse graphs. Another is that of determining induced Ramsey numbers, i.e., proving, for any given $H$, that there is a graph $G$ such that any two-coloring of the edges of $G$ contains an induced

\footnotetext{
*St John's College, Cambridge, United Kingdom. E-mail: D.Conlon@dpmms.cam.ac.uk. Research supported by a Junior Research Fellowship at St John's College.

${ }^{\dagger}$ Department of Mathematics, Princeton, Princeton, NJ. Email: jacobfox@math.princeton.edu. Research supported by an NSF Graduate Research Fellowship and a Princeton Centennial Fellowship.

${ }^{\ddagger}$ Department of Mathematics, UCLA, Los Angeles, CA 90095. Email: bsudakov@math.ucla.edu. Research supported in part by NSF CAREER award DMS-0812005 and by a USA-Israeli BSF grant.
} 
monochromatic copy of $H$. In this paper, we present a unified approach which allows us to make improvements to two classical questions in these areas.

In 1975, Burr and Erdős [2] posed the problem of showing that every graph $H$ with $n$ vertices and maximum degree $\Delta$ satisfied $r(H) \leq c(\Delta) n$, where the constant $c(\Delta)$ depends only on $\Delta$. That this is indeed the case was shown by Chvátal, Rödl, Szemerédi and Trotter [4] in one of the earliest applications of Szemerédi's celebrated regularity lemma [34]. Remarkably, this means that for graphs of fixed maximum degree the Ramsey number only has a linear dependence on the number of vertices. Unfortunately, because it uses the regularity lemma, the bounds that the original method gives on $c(\Delta)$ are (and are necessarily [21]) of tower type in $\Delta$. More precisely, $c(\Delta)$ works out as being an exponential tower of $2 \mathrm{~s}$ with a height that is itself exponential in $\Delta$.

The situation was remedied somewhat by Eaton [11, who proved, using a variant of the regularity lemma, that the function $c(\Delta)$ can be taken to be of the form $2^{2^{c \Delta}}$. Soon after, Graham, Rödl and Ruciński proved [22, by a beautiful method which avoids any use of the regularity lemma, that there exists a constant $c$ for which

$$
c(\Delta) \leq 2^{c \Delta \log ^{2} \Delta} .
$$

For bipartite graphs, they were able to do even better [23], showing that if $H$ is a bipartite graph with $n$ vertices and maximum degree $\Delta$ then $r(H) \leq 2^{c \Delta \log \Delta} n$. They also proved that there are bipartite graphs with $n$ vertices and maximum degree $\Delta$ for which the Ramsey number is at least $2^{c^{\prime} \Delta} n$. Recently, Conlon [6] and, independently, Fox and Sudakov [19] have shown how to remove the $\log \Delta$ factor in the exponent, achieving an essentially best possible bound of $r(H) \leq 2^{c \Delta} n$ in the bipartite case. These results were jointly extended to hypergraphs in [7], after several proofs [8, 9, 29] using the hypergraph regularity lemma.

Unfortunately, if one tries to use these recent techniques to treat general graphs, the best one seems to be able to achieve is $c(\Delta) \leq 2^{c \Delta^{2}}$. In this paper we take a different approach, more closely related to that of Graham, Rödl and Ruciński [22]. Improving on their bound, we show that $c(\Delta) \leq 2^{c \Delta \log \Delta}$, which brings us a step closer to matching the lower bound of $2^{c^{\prime} \Delta}$.

Theorem 1.1 There exists a constant $c$ such that, for every graph $H$ with $n$ vertices and maximum degree $\Delta$,

$$
r(H) \leq 2^{c \Delta \log \Delta} n
$$

A graph $H$ is said to be an induced subgraph of $H$ if $V(H) \subset V(G)$ and two vertices of $H$ are adjacent if and only if they are adjacent in $G$. The induced Ramsey number $r_{i n d}(H)$ is the smallest natural number $N$ for which there is a graph $G$ on $N$ vertices such that in every two-coloring of the edges of $G$ there is an induced monochromatic copy of $H$. The existence of these numbers was independently proven by Deuber [10], Erdös, Hajnal and Pósa [16] and Rödl [31. The bounds that these original proofs give on $r_{\text {ind }}(H)$ are enormous, but it was conjectured by Erdös [13] that the actual values should be more in line with ordinary Ramsey numbers. More specifically, he conjectured the existence of a constant $c$ such that every graph $H$ with $n$ vertices satisfies $r_{i n d}(H) \leq 2^{c n}$. If true, the complete graph shows that it would be best possible.

In a problem paper, Erdős [12] stated that he and Hajnal had proved a bound of the form $r_{\text {ind }}(H) \leq$ $2^{2^{n^{1+o(1)}}}$. This remained the state of the art for some years until Kohayakawa, Prömel and Rödl [25] proved that there was a constant $c$ such that every graph $H$ on $n$ vertices satisfies $r_{i n d}(H) \leq 2^{c n \log ^{2} n}$. As in the bounded-degree problem, we remove one of the logarithms in the exponent. 
Theorem 1.2 There exists a constant $c$ such that every graph $H$ with $n$ vertices satisfies

$$
r_{\text {ind }}(H) \leq 2^{c n \log n} .
$$

It is worth noting that the graph $G$ that Kohayakawa, Prömel and Rödl use in their proofs is a random graph constructed with projective planes. This graph is specifically designed so as to contain many copies of our target graph $H$. Recently, Fox and Sudakov [18] showed how to prove the same bounds as Kohayakawa, Prömel and Rödl using explicit pseudo-random graphs. We will follow a similar path.

A graph is said to be pseudo-random if it imitates some of the properties of a random graph. One such random-like property, introduced by Thomason [35, 36], is that of having approximately the same density between any pair of large disjoint vertex sets. More formally, we say that a graph $G=(V, E)$ is $(p, \lambda)$-pseudo-random if, for all subsets $A, B$ of $V$, the density of edges $d(A, B)$ between $A$ and $B$ satisfies

$$
|d(A, B)-p| \leq \frac{\lambda}{\sqrt{|A||B|}} .
$$

The usual random graph $G(N, p)$, where each edge is chosen independently with probability $p$, is itself a $(p, \lambda)$-pseudo-random graph where $\lambda$ is on the order of $\sqrt{N}$. A well-known explicit example, known to be $\left(\frac{1}{2}, \sqrt{N}\right)$-pseudo-random, is the Paley graph $P_{N}$. This graph is defined by setting $V$ to be the set $\mathbb{Z}_{N}$, where $N$ is a prime which is congruent to 1 modulo 4 , and taking two vertices $x, y \in V$ to be adjacent if and only if $x-y$ is a quadratic residue. For further information on this and other pseudorandom graphs we refer the reader to [27]. Our next theorem states that, for $\lambda$ sufficiently small, a $\left(\frac{1}{2}, \lambda\right)$-pseudo-random graph has very strong Ramsey properties. Theorem 1.2 follows by applying this theorem to the particular examples of pseudo-random graphs given above.

Theorem 1.3 There exists a constant $c$ such that, for any $n \in \mathbb{N}$ and any $\left(\frac{1}{2}, \lambda\right)$-pseudo-random graph $G$ on $N$ vertices with $\lambda \leq 2^{-c n \log n} N$, every graph on $n$ vertices occurs as an induced monochromatic copy in all 2-edge-colorings of $G$. Moreover, all of these induced monochromatic copies can be found in the same color.

The theme that unites these two, apparently disparate, questions is the method we employ in our proofs. A simplified version of this method is the following. In the first color we attempt to find a large subset in which this color is very dense. If such a set can be found, we can easily embed the required graph. If, on the other hand, this is not the case, then there is a large subset in which the edges of the second color are well-distributed. Again, this allows us to prove an embedding lemma. Such ideas are already explicit in the work of Graham, Rödl and Ruciński and, arguably, implicit in that of Kohayakawa, Prömel and Rödl. The advantage of our method, which extends upon these ideas, is that it is much more symmetrical between the colors. It is this symmetry which allows us to drop a log factor in each case.

In the next section, we will prove Theorem 1.1. Section 3 contains the proof of Theorem 1.3. The last section contains some concluding remarks together with a discussion of a few conjectures and open problems. Throughout the paper, we systematically omit floor and ceiling signs whenever they are not crucial for the sake of clarity of presentation. All logarithms, unless otherwise stated, are to the base 2. We also do not make any serious attempt to optimize absolute constants in our statements and proofs. 


\section{Ramsey number of bounded-degree graphs}

The edge density $d(X, Y)$ between two disjoint vertex subsets $X, Y$ of a graph $G$ is the fraction of pairs $(x, y) \in X \times Y$ that are edges of $G$. That is, $d(X, Y)=\frac{e(X, Y)}{|X||Y|}$, where $e(X, Y)$ is the number of edges with one endpoint in $X$ and the other in $Y$. In a graph $G$, a vertex subset $U$ is called $b i$ - $(\epsilon, \rho)$-dense if, for all disjoint pairs $A, B \subset U$ with $|A|,|B| \geq \epsilon|U|$, we have $d(A, B) \geq \rho$. We call a graph $G$ bi- $(\epsilon, \rho)$-dense if its vertex set $V(G)$ is bi- $(\epsilon, \rho)$-dense. Trivially, if $\epsilon^{\prime} \leq \epsilon$ and $\rho^{\prime} \geq \rho$, then a bi- $\left(\epsilon^{\prime}, \rho^{\prime}\right)$-dense graph is also bi- $(\epsilon, \rho)$-dense. Moreover, if $\epsilon>1 / 2$, then every graph is vacuously bi- $(\epsilon, \rho)$-dense as there is no pair of disjoint subsets each with more than half of the vertices.

Before going into the proof of Theorem 1.1, we first sketch for comparison the original idea of Graham, Rödl, and Rucinski [22] which gives a weaker bound. We then discuss our proof technique. They noticed that if a graph $G$ on $N$ vertices is bi- $(\epsilon, \rho)$-dense with $\epsilon=\rho^{\Delta} /(\Delta+1)$ and $N \geq 2 \rho^{-\Delta}(\Delta+1) n$, then $G$ contains every $n$-vertex graph $H$ of maximum degree $\Delta$. This can be shown by embedding $H$ one vertex at a time. In particular, if a red-blue edge-coloring of $K_{N}$ does not contain a monochromatic copy of $H$, then the red graph is not bi- $(\epsilon, \rho)$-dense, and there are disjoint vertex subsets $A$ and $B$ with $|A|,|B| \geq \epsilon N$ such that the red density between them at most $\rho$. It is then possible to iterate, at the expense of another factor in the exponent of roughly $\log (1 / \rho)$, to get a subset $S$ of size roughly $\epsilon^{\log (1 / \rho)} N$ with red edge density at most $2 \rho$ inside. Picking $\rho=\frac{1}{16 \Delta}$, a simple greedy embedding then shows that inside $S$ we can find a blue copy of any graph with at most $|S| / 4$ vertices and maximum degree $\Delta$.

To summarize, the proof finds a vertex subset $S$ which is either bi- $(\epsilon, \rho)$-dense in the red graph or is very dense in the blue graph. In either case, it is easy to find a monochromatic copy of any $n$-vertex graph $H$ with maximum degree $\Delta$.

We will instead find a sequence of large vertex subsets $S_{1}, \ldots, S_{t}$ such that, in one of the two colors, each of the subsets satisfies some bi-density condition and the graph between these subsets is very dense. The bi-density condition inside each $S_{i}$ is roughly the condition which ensures that we can embed any graph on $n$ vertices with maximum degree $d_{i}$, where $d_{1}+\ldots+d_{t}=\Delta-t+1$. A simple lemma of Lovász guarantees that we can partition $V(H)=V_{1} \cup \ldots \cup V_{t}$ such that the induced subgraph of $H$ with vertex set $V_{i}$ has maximum degree at most $d_{i}$. Our embedding lemma shows that we can embed a monochromatic copy of $H$ with the image of $V_{i}$ being in $S_{i}$. We now proceed to the details of the proof.

Definition: A graph on $N$ vertices is $(\alpha, \beta, \rho, \Delta)$-dense if there is a sequence $S_{1}, \ldots, S_{t}$ of disjoint vertex subsets each of cardinality at least $\alpha N$ and nonnegative integers $d_{1}, \ldots, d_{t}$ such that $d_{1}+\cdots+$ $d_{t}=\Delta-t+1$, and the following holds:

- for $1 \leq i \leq t, S_{i}$ is $\operatorname{bi}-\left(\rho^{2 d_{i}}, \rho\right)$-dense, and

- for $1 \leq i<j \leq t$, each vertex in $S_{i}$ has at least $(1-\beta)\left|S_{j}\right|$ neighbors in $S_{j}$.

Note that since $d_{1}+\cdots+d_{t}=\Delta-t+1$ and each $d_{i}$ is nonnegative, we must have $t \leq \Delta+1$.

Trivially, if a graph is $\left(\alpha^{\prime}, \beta^{\prime}, \rho, \Delta^{\prime}\right)$-dense and $\alpha^{\prime} \geq \alpha, \beta^{\prime} \leq \beta$, and $\Delta^{\prime} \geq \Delta$, then it is also $(\alpha, \beta, \rho, \Delta)$ dense.

We say a red-blue edge-coloring of the complete graph $K_{N}$ is $\left(\alpha, \beta, \rho, \Delta_{1}, \Delta_{2}\right)$-dense if the red graph is $\left(\alpha, \beta, \rho, \Delta_{1}\right)$-dense or the blue graph is $\left(\alpha, \beta, \rho, \Delta_{2}\right)$-dense. We say that $\left(\alpha, \beta, \rho, \Delta_{1}, \Delta_{2}\right)$ is universal if, for every $N$, every red-blue edge-coloring of $K_{N}$ is $\left(\alpha, \beta, \rho, \Delta_{1}, \Delta_{2}\right)$-dense. 
Lemma 2.1 If $\beta \geq 4\left(\Delta_{2}+1\right) \rho$ and $\left(\alpha, \beta, \rho, \Delta_{1}, \Delta_{2}\right)$ is universal, then $\left(\frac{1}{2} \rho^{2 \Delta_{1}} \alpha, \beta, \rho, \Delta_{1}, 2 \Delta_{2}+1\right)$ is also universal.

Proof: Consider a red-blue edge-coloring of a complete graph $K_{N}$. If the red graph is bi- $\left(\rho^{2 \Delta_{1}}, \rho\right)$ dense, then, taking $t=1, S_{1}=V\left(K_{N}\right)$ and $d_{1}=\Delta_{1}$, we see that the red graph is $\left(\alpha, \beta, \rho, \Delta_{1}\right)$-dense and we are done. So we may suppose that there are disjoint vertex subsets $V_{0}, V_{1}$ with $\left|V_{0}\right|,\left|V_{1}\right| \geq$ $\rho^{2 \Delta_{1}} N$ such that the red density between them is less than $\rho$. Delete from $V_{0}$ all vertices in at least $2 \rho\left|V_{1}\right|$ red edges with vertices in $V_{1}$; the remaining subset $V_{0}^{\prime}$ has cardinality at least $\frac{1}{2}\left|V_{0}\right| \geq \frac{1}{2} \rho^{2 \Delta_{1}} N$. Since $\left(\alpha, \beta, \rho, \Delta_{1}, \Delta_{2}\right)$ is universal, the coloring restricted to $V_{0}^{\prime}$ is $\left(\alpha, \beta, \rho, \Delta_{1}, \Delta_{2}\right)$-dense. Thus, the red graph is $\left(\alpha, \beta, \rho, \Delta_{1}\right)$-dense (in which case we are again done) or the blue graph is $\left(\alpha, \beta, \rho, \Delta_{2}\right)$ dense. We may suppose the latter holds, and there are subsets $S_{1}, \ldots, S_{t}$ each of cardinality at least $\alpha\left|V_{0}^{\prime}\right| \geq \frac{1}{2} \rho^{2 \Delta_{1}} \alpha N$ and nonnegative integers $d_{1}, \ldots, d_{t}$ such that $d_{1}+\cdots+d_{t}=\Delta_{2}-t+1$, and the following holds:

- for $1 \leq i \leq t, S_{i}$ is $\operatorname{bi}-\left(\rho^{2 d_{i}}, \rho\right)$-dense, and

- for $1 \leq i<j \leq t$, each vertex in $S_{i}$ has at least $(1-\beta)\left|S_{j}\right|$ neighbors in $S_{j}$.

Since each vertex in $V_{0}^{\prime}$ (and hence in each $S_{i}$ ) is in at most $2 \rho\left|V_{1}\right|$ red edges with vertices in $V_{1}$, there are at most $2 \rho\left|S_{i}\right|\left|V_{1}\right|$ red edges between $S_{i}$ and $V_{1}$. For $1 \leq i \leq t$, delete from $V_{1}$ all vertices in at least $4\left(\Delta_{2}+1\right) \rho\left|S_{i}\right|$ red edges with vertices in $S_{i}$. For any given $i$, there can be at most $\frac{1}{2\left(\Delta_{2}+1\right)}\left|V_{1}\right|$ such vertices. Therefore, since $t \leq \Delta_{2}+1$, the set $V_{1}^{\prime}$ of remaining vertices has cardinality at least $\left|V_{1}\right|-t \cdot \frac{1}{2\left(\Delta_{2}+1\right)}\left|V_{1}\right| \geq\left|V_{1}\right| / 2$.

Since $\left(\alpha, \beta, \rho, \Delta_{1}, \Delta_{2}\right)$ is universal, the coloring restricted to $V_{1}^{\prime}$ is $\left(\alpha, \beta, \rho, \Delta_{1}, \Delta_{2}\right)$-dense. Thus, the red graph is $\left(\alpha, \beta, \rho, \Delta_{1}\right)$-dense (in which case we are done) or the blue graph is $\left(\alpha, \beta, \rho, \Delta_{2}\right)$-dense. We may suppose the latter holds, and there are subsets $T_{1}, \ldots, T_{u}$ each of cardinality at least $\alpha\left|V_{1}^{\prime}\right| \geq \frac{1}{2} \rho^{2 \Delta_{1}} \alpha N$ and nonnegative integers $e_{1}, \ldots, e_{u}$ such that $e_{1}+\cdots+e_{u}=\Delta_{2}-u+1$, and the following holds:

- for $1 \leq i \leq u, T_{i}$ is $\operatorname{bi}-\left(\rho^{2 e_{i}}, \rho\right)$-dense, and

- for $1 \leq i<j \leq u$, each vertex in $T_{i}$ has at least $(1-\beta)\left|T_{j}\right|$ neighbors in $T_{j}$.

Note that $e_{1}+\cdots+e_{u}+d_{1}+\cdots+d_{t}=\Delta_{2}-u+1+\Delta_{2}-t+1=\left(2 \Delta_{2}+1\right)-(u+t)+1$. Moreover, $\beta \geq 4\left(\Delta_{2}+1\right) \rho$, implying that for all $1 \leq i \leq u$ and all $1 \leq j \leq t$ every vertex in $T_{i}$ has at least $(1-\beta)\left|S_{j}\right|$ neighbors in $S_{j}$. Therefore, the sequence $T_{1}, \ldots, T_{u}, S_{1}, \ldots, S_{t}$ implies that the blue graph is $\left(\frac{1}{2} \rho^{2 \Delta_{1}} \alpha, \beta, \rho, 2 \Delta_{2}+1\right)$-dense, completing the proof.

By symmetry, the above lemma implies that if $\beta \geq 4\left(\Delta_{1}+1\right) \rho$ and $\left(\alpha, \beta, \rho, \Delta_{1}, \Delta_{2}\right)$ is universal, then $\left(\frac{1}{2} \rho^{2 \Delta_{2}} \alpha, \beta, \rho, 2 \Delta_{1}+1, \Delta_{2}\right)$ is also universal.

As already mentioned, if $\epsilon>1 / 2$, every graph $G$ is vacuously bi- $(\epsilon, \rho)$-dense. As $\rho^{2 \cdot 0}=1>1 / 2$, setting $t=1$ and $S_{1}=V(G)$, we have that every graph $G$ is $(\alpha, \beta, \rho, 0)$-dense. This shows that $(1,2 \rho, \rho, 0,0)$ is universal, which is the base case $h=0$ in the induction proof of the next lemma.

Lemma 2.2 Let $h$ be a nonnegative integer and $D:=2^{h}-1$. Then $\left(2^{-2 h} \rho^{6 D-4 h}, 2(D+1) \rho, \rho, D, D\right)$ is universal. 
Proof: As mentioned above, the proof is by induction on $h$, and the base case $h=0$ is satisfied. Suppose it is satisfied for $h$, and we wish to show it for $h+1$. Let $D=2^{h}-1, D^{\prime}=2 D+1=2^{h+1}-1$, and $\beta=4(D+1) \rho=2\left(D^{\prime}+1\right) \rho \geq 2(D+1) \rho$. Recall that, for $\beta \geq \beta^{\prime}$, if $\left(\alpha, \beta^{\prime}, \rho, \Delta_{1}, \Delta_{2}\right)$ is universal then so is $\left(\alpha, \beta, \rho, \Delta_{1}, \Delta_{2}\right)$. Therefore, since $\left(2^{-2 h} \rho^{6 D-4 h}, 2(D+1) \rho, \rho, D, D\right)$ is universal, $\left(2^{-2 h} \rho^{6 D-4 h}, \beta, \rho, D, D\right)$ is also. Applying Lemma 2.1, we have that $\left(\frac{1}{2} \rho^{2 D} 2^{-2 h} \rho^{6 D-4 h}, \beta, \rho, D, 2 D+1\right)$ is universal. Applying the symmetric version of Lemma 2.1 mentioned above, we have that

$$
\left(\frac{1}{2} \rho^{2(2 D+1)} \frac{1}{2} \rho^{2 D} 2^{-2 h} \rho^{6 D-4 h}, \beta, \rho, 2 D+1,2 D+1\right)=\left(2^{-2(h+1)} \rho^{6 D^{\prime}-4(h+1)}, \beta, \rho, D^{\prime}, D^{\prime}\right),
$$

is universal, which completes the proof by induction.

We will use the following lemma of Lovász [28].

Lemma 2.3 If $H$ has maximum degree $\Delta$ and $d_{1}, \ldots, d_{t}$ are nonnegative integers satisfying $d_{1}+\cdots+$ $d_{t}=\Delta-t+1$, then there is a partition $V(H)=V_{1} \cup \ldots \cup V_{t}$ such that for $1 \leq i \leq t$, the induced subgraph of $H$ with vertex set $V_{i}$ has maximum degree at most $d_{i}$.

The next simple lemma shows that in a bi- $(\epsilon, \rho)$-dense graph, for any large vertex subset $B$, there are few vertices with few neighbors in $B$.

Lemma 2.4 If $G$ is a bi- $(\epsilon, \rho)$-dense graph on $n$ vertices with $\epsilon \geq 1 / n$ and $B \subset V(G)$ with $|B| \geq 2 \epsilon n$, then there are less than $3 \epsilon n$ vertices in $G$ with fewer than $\frac{\rho}{2}|B|$ neighbors in $B$.

Proof: Suppose for contradiction that the set $A$ of vertices in $G$ with fewer than $\frac{\rho}{2}|B|$ neighbors in $B$ satisfies $|A| \geq 3 \epsilon n$. Partition $A \cap B=C_{1} \cup C_{2}$ with $\left|C_{1}\right| \leq\left|C_{2}\right|$ into two sets of size as equal as possible. Then the sets $A^{\prime}=A \backslash C_{2}$ and $B^{\prime}=B \backslash C_{1}$ are disjoint, $\left|A^{\prime}\right| \geq\lfloor|A| / 2\rfloor \geq \epsilon n,\left|B^{\prime}\right| \geq|B| / 2 \geq \epsilon n$, the number of edges between $A^{\prime}$ and $B^{\prime}$ is less than $\left|A^{\prime}\right| \frac{\rho}{2}|B|$, and the edge density between $A^{\prime}$ and $B^{\prime}$ is less than $\frac{\left|A^{\prime}\right| \frac{\rho}{2}|B|}{\left|A^{\prime}\right|\left|B^{\prime}\right|}=\frac{\rho}{2} \frac{|B|}{\left|B^{\prime}\right|} \leq \rho$, contradicting $G$ is bi- $(\epsilon, \rho)$-dense.

The following embedding lemma is the last ingredient for the proof of Theorem 1.1 .

Lemma 2.5 If $\rho \leq 1 / 30$ and $G$ is a graph on $N \geq 4(2 / \rho)^{2 \Delta} \alpha^{-1} n$ vertices which is $\left(\alpha, \frac{1}{2 \Delta}, \rho, \Delta\right)$-dense, then $G$ contains every graph $H$ on $n$ vertices with maximum degree at most $\Delta$.

Proof: Since $G$ is $\left(\alpha, \frac{1}{2 \Delta}, \rho, \Delta\right)$-dense, there is a sequence $S_{1}, \ldots, S_{t}$ of disjoint vertex subsets each of cardinality at least $\alpha N$ and nonnegative integers $d_{1}, \ldots, d_{t}$ such that $d_{1}+\cdots+d_{t}=\Delta-t+1$, and the following holds:

- for $1 \leq i \leq t, S_{i}$ is $\operatorname{bi}-\left(\rho^{2 d_{i}}, \rho\right)$-dense, and

- for $1 \leq i<j \leq t$, each vertex in $S_{i}$ has at least $\left(1-\frac{1}{2 \Delta}\right)\left|S_{j}\right|$ neighbors in $S_{j}$.

By Lemma 2.3. there is a vertex partition $V(H)=V_{1} \cup \ldots \cup V_{t}$ such that the maximum degree of the induced subgraph of $H$ with vertex set $V_{i}$ is at most $d_{i}$ for $1 \leq i \leq t$. Let $v_{1}, \ldots, v_{n}$ be an ordering of the vertices in $V(H)$ such that the vertices in $V_{i}$ come before the vertices in $V_{j}$ for $i<j$. Let $N(h, k)$ denote the set of neighbors $v_{i}$ of $v_{k}$ with $i \leq h$. For $v_{k} \in V_{j}$, let $M(h, k)$ denote the set of neighbors 
$v_{i} \in V_{j}$ of $v_{k}$ with $i \leq h$, that is, $M(h, k)=N(h, k) \cap V_{j}$. Notice that $|M(h, k)| \leq d_{j}$ for $v_{k} \in V_{j}$ since the induced subgraph of $H$ with vertex set $V_{j}$ has maximum degree at most $d_{j}$.

We will find an embedding $f: V(H) \rightarrow V(G)$ of $H$ in $G$ such that $f\left(V_{i}\right) \subset S_{i}$ for each $i$. We will embed the vertices in increasing order of their indices. The embedding will have the property that after embedding the first $h$ vertices, if $k>h$ and $v_{k} \in V_{j}$, then the set $S(h, k)$ of vertices in $S_{j}$ adjacent to all vertices in $f(N(h, k))$ has cardinality at least $\frac{1}{2}(\rho / 2)^{|M(h, k)|}\left|S_{j}\right|$. Notice that this condition is trivially satisfied when $h=0$. Suppose that this condition is satisfied after embedding the first $h$ vertices. The set $S(h, k)$ are the potential vertices in which to embed $v_{k}$ after the first $h$ vertices have been embedded, though this set may already contain embedded vertices.

Let $j$ be such that $v_{h+1} \in V_{j}$. We need to find a vertex in $S(h, h+1)$ to embed the copy of $v_{h+1}$. We have

$$
|S(h, h+1)| \geq \frac{1}{2}(\rho / 2)^{|M(h, h+1)|}\left|S_{j}\right| \geq \frac{1}{2}(\rho / 2)^{d_{j}}\left|S_{j}\right|
$$

since $|M(h, h+1)| \leq d_{j}$. If $d_{j}=0$, we may pick $f\left(v_{h+1}\right)$ to be any element of the set $S(h, h+$ $1) \backslash\left\{f\left(v_{1}\right), \ldots, f\left(v_{h}\right)\right\}$. We may assume, therefore, that $1 \leq d_{j} \leq \Delta$. In this case we know, for each of the at most $d_{j}$ neighbors $v_{k}$ of $v_{h+1}$ with $k>h+1$ that are in $V_{j}$, that the set $S(h, k)$ has cardinality at least $\frac{1}{2}(\rho / 2)^{d_{j}}\left|S_{j}\right|$. Let $\epsilon=\rho^{2 d_{j}}$. Since, for $1 \leq d_{j} \leq \Delta$ and $\rho \leq 1 / 30, S_{j}$ is bi- $\left(\rho^{2 d_{j}}, \rho\right)$-dense, $|S(h, k)| \geq \frac{1}{2}(\rho / 2)^{d_{j}}\left|S_{j}\right| \geq 2 \rho^{2 d_{j}}\left|S_{j}\right|=2 \epsilon\left|S_{j}\right|$ and $\epsilon\left|S_{j}\right|=\rho^{2 d_{j}}\left|S_{j}\right| \geq \rho^{2 \Delta} \alpha N \geq 1$, we may apply Lemma 2.4 in $S_{j}$ with $B=S(h, k)$. Therefore, for each vertex $v_{k} \in V_{j}, k>h+1$ adjacent to $v_{h+1}$, at most $3 \rho^{2 d_{j}}\left|S_{j}\right|$ vertices in $S_{j}$ have fewer than $\frac{\rho}{2}|S(h, k)|$ neighbors in $S(h, k)$. Thus, all but at most $d_{j} \cdot 3 \rho^{2 d_{j}}\left|S_{j}\right|$ vertices in $S_{j}$ have at least $\frac{\rho}{2}|S(h, k)|$ neighbors in $S(h, k)$ for all $v_{k} \in V_{j}, k>h+1$ that are neighbors of $v_{h+1}$. Since, for $\rho \leq 1 / 30$, we have $d_{j} \cdot 3 \rho^{2 d_{j}} \leq \frac{1}{4}(\rho / 2)^{d_{j}}$, there are at least

$$
\begin{aligned}
|S(h, h+1)|-d_{j} \cdot 3 \rho^{2 d_{j}}\left|S_{j}\right|-h & \geq \frac{1}{2}(\rho / 2)^{d_{j}}\left|S_{j}\right|-d_{j} \cdot 3 \rho^{2 d_{j}}\left|S_{j}\right|-h \geq \frac{1}{4}(\rho / 2)^{d_{j}}\left|S_{j}\right|-h \\
& \geq \frac{1}{4}(\rho / 2)^{\Delta} \alpha N-h \geq(2 / \rho)^{\Delta} n-h>0
\end{aligned}
$$

such vertices that are not already embedded. We can pick any of these vertices to be $f\left(v_{h+1}\right)$. To continue, it remains to check that any such choice preserves the properties of our embedding. Indeed,

- for any $k<h+1$ for which $v_{h+1}$ is adjacent to $v_{k}, f\left(v_{h+1}\right)$ is adjacent to $f\left(v_{k}\right)$;

- if $k>h+1$ and $v_{k}$ and $v_{h+1}$ are not adjacent, then $S(h+1, k)=S(h, k)$ and $M(h+1, k)=$ $M(h, k)$;

- if, for some $k>h+1, v_{k}$ and $v_{h+1}$ are adjacent and $v_{k} \in V_{\ell}$ with $\ell \neq j$, then $M(h+1, k)=0$ since vertices of $V_{j}$ are embedded before vertices of $V_{\ell}, \ell>j$, so no vertex of $V_{\ell}$ was embedded yet. Also, $|S(h+1, k)| \geq \frac{1}{2}\left|S_{\ell}\right|$ since $|N(h+1, k)| \leq \Delta$, the vertices in $f(N(h+1, k))$ each have at least $\left(1-\frac{1}{2 \Delta}\right)\left|S_{\ell}\right|$ neighbors in $S_{\ell}$, and hence $|S(h+1, k)| \geq\left|S_{\ell}\right|-\Delta \cdot \frac{1}{2 \Delta}\left|S_{\ell}\right|=\frac{1}{2}\left|S_{\ell}\right|$;

- if $k>h+1, v_{k}$ and $v_{h+1}$ are adjacent and $v_{k} \in V_{j}$, then $|M(h+1, k)|=|M(h, k)|+1$. Moreover, by our choice of the vertex $f\left(v_{h+1}\right)$, it has at least $\frac{\rho}{2}|S(h, k)|$ neighbors in $S(h, k)$. Therefore $|S(h+1, k)| \geq \frac{\rho}{2}|S(h, k)| \geq \frac{1}{2}(\rho / 2)^{|M(h, k)|+1}\left|S_{j}\right|=\frac{1}{2}(\rho / 2)^{|M(h+1, k)|}\left|S_{j}\right|$, as required.

As we supposed there is an embedding of the first $h$ vertices with the desired property, the above four facts imply that there is an embedding of the first $h+1$ vertices with the desired property. By induction on $h$, we find an embedding of $H$ in $G$.

We can now prove the following theorem, which implies Theorem 1.1. 
Theorem 2.1 For every 2-edge-coloring of $K_{N}$ with $N=2^{84 \Delta+2} \Delta^{32 \Delta} n$, at least one of the color classes contains a copy of every graph on $n$ vertices with maximum degree $\Delta \geq 2$.

Proof: Let $h$ be the smallest positive integer such that $D:=2^{h}-1 \geq \Delta$. By the definition of $D$, $\Delta \leq D<2 \Delta$. Let $\rho=\frac{1}{8 D^{2}}, \alpha=2^{-2 h} \rho^{6 D-4 h} \geq \rho^{6 D}$, and $\beta=2(D+1) \rho \leq \frac{1}{2 D}$. Lemma 2.2 implies that every red-blue coloring of the edges of the complete graph $K_{N}$ is $(\alpha, \beta, \rho, D, D)$-dense. By Lemma 2.5. since

$$
\begin{aligned}
4(2 / \rho)^{2 D} \alpha^{-1} n & \leq 4\left(16 D^{2}\right)^{2 D} \cdot\left(8 D^{2}\right)^{6 D} n \leq 4\left(16(2 \Delta)^{2}\right)^{4 \Delta}\left(8(2 \Delta)^{2}\right)^{12 \Delta} n \\
& =2^{2}\left(2^{6} \Delta^{2}\right)^{4 \Delta}\left(2^{5} \Delta^{2}\right)^{12 \Delta} n=2^{84 \Delta+2} \Delta^{32 \Delta} n=N
\end{aligned}
$$

at least one of the color classes contains a copy of every graph on $n$ vertices with maximum degree $\Delta$.

\section{Induced Ramsey numbers}

The goal of this section is to prove Theorem 1.3. We will do this by finding, in any 2-edge-coloring of the pseudo-random graph $G$, a collection of vertex subsets $S_{1}, \ldots, S_{t}$ satisfying certain conditions. The conditions in question are closely related to the notion of density that we applied in the last section. Now, as then, we demand that the graph of one particular color satisfies a certain bi-density condition within each $S_{i}$. In addition, we demand that between the different $S_{i}$ the other color be sparse. This may look like a simple rearrangement of the condition from the previous section, but, given that we are now looking at colorings of a pseudo-random graph $G$ rather than the complete graph $K_{N}$, the condition is more general. Moreover, it is exactly what we need to make our embedding lemma work.

Definition: An edge-coloring of a graph $G$ on $N$ vertices with colors 1 and 2 is $\left(\alpha, \beta, \rho, f, \Delta_{1}, \Delta_{2}\right)$ dense if there is a color $q \in\{1,2\}$, disjoint vertex subsets $S_{1}, \ldots, S_{t}$ each of cardinality at least $\alpha N$ and nonnegative integers $d_{1}, \ldots, d_{t}$ with $d_{1}+\cdots+d_{t}=\Delta_{q}-t+1$ such that the following holds:

- for $1 \leq i \leq t, S_{i}$ is bi- $\left(f\left(\rho, d_{i}\right), \rho\right)$-dense in the graph of color $q$, and

- for $1 \leq i<j \leq t$, each vertex in $S_{i}$ is in at most $\beta\left|S_{j}\right|$ edges of color $3-q$ with vertices in $S_{j}$.

We say that $\left(\alpha, \beta, \rho, f, \Delta_{1}, \Delta_{2}\right)$ is universal if, for every graph $G$, every edge-coloring of $G$ with colors 1 and 2 is $\left(\alpha, \beta, \rho, f, \Delta_{1}, \Delta_{2}\right)$-dense. Note that the density condition used in the last section corresponds to the case when $G=K_{N}$ and $f\left(\rho, d_{i}\right)=\rho^{2 d_{i}}$. Essentially the same proofs as Lemmas 2.1 and 2.2 give the following two more general lemmas. We include the proofs for completeness.

Lemma 3.1 If $\beta \geq 4\left(\Delta_{2}+1\right) \rho$ and $\left(\alpha, \beta, \rho, f, \Delta_{1}, \Delta_{2}\right)$ is universal, then $\left(\frac{1}{2} f\left(\rho, \Delta_{1}\right) \alpha, \beta, \rho, f, \Delta_{1}, 2 \Delta_{2}+\right.$ 1) is also universal.

Proof: Consider an edge-coloring of a graph $G$ with colors 1 and 2. If the graph of color 1 is bi$\left(f\left(\rho, \Delta_{1}\right), \rho\right)$-dense, then, taking, $q=1, t=1, S_{1}=V(G)$ and $d_{1}=\Delta_{1}$, we are done. So we may suppose that there are disjoint vertex subsets $V_{0}, V_{1}$ with $\left|V_{0}\right|,\left|V_{1}\right| \geq f\left(\rho, \Delta_{1}\right) N$ such that the density of color 1 between them is less than $\rho$. Delete from $V_{0}$ all vertices in at least $2 \rho\left|V_{1}\right|$ edges of color 
1 with vertices in $V_{1}$; the remaining subset $V_{0}^{\prime}$ has cardinality at least $\frac{1}{2}\left|V_{0}\right| \geq \frac{1}{2} f\left(\rho, \Delta_{1}\right) N$. Since $\left(\alpha, \beta, \rho, f, \Delta_{1}, \Delta_{2}\right)$ is universal, the coloring restricted to the induced subgraph of $G$ with vertex set $V_{0}^{\prime}$ is $\left(\alpha, \beta, \rho, f, \Delta_{1}, \Delta_{2}\right)$-dense. Thus, there is $q \in\{1,2\}$, disjoint vertex subsets $S_{1}, \ldots, S_{t} \subset V_{0}^{\prime}$ each of cardinality at least $\alpha\left|V_{0}^{\prime}\right|$ and nonnegative integers $d_{1}, \ldots, d_{t}$ with $d_{1}+\cdots+d_{t}=\Delta_{q}-t+1$ such that the following holds:

- for $1 \leq i \leq t, S_{i}$ is bi- $\left(f\left(\rho, d_{i}\right), \rho\right)$-dense in the graph of color $q$, and

- for $1 \leq i<j \leq t$, each vertex in $S_{i}$ is in at most $\beta\left|S_{j}\right|$ edges of color $3-q$ with vertices in $S_{j}$.

If $q=1$, we are done. Therefore, we may suppose $q=2$.

Since each vertex in $V_{0}^{\prime}$ (and hence in each $S_{i}$ ) is in at most $2 \rho\left|V_{1}\right|$ edges of color 1 with vertices in $V_{1}$, then there are at most $2 \rho\left|S_{i}\right|\left|V_{1}\right|$ edges of color 1 between $S_{i}$ and $V_{1}$. For $1 \leq i \leq t$, delete from $V_{1}$ all vertices in at least $4\left(\Delta_{2}+1\right) \rho\left|S_{i}\right|$ edges of color 1 with vertices in $S_{i}$. For any given $i$, there can be at most $\frac{1}{2\left(\Delta_{2}+1\right)}\left|V_{1}\right|$ such vertices. Therefore, since $t \leq \Delta_{2}+1$, the set $V_{1}^{\prime}$ of remaining vertices has cardinality at least $\left|V_{1}\right|-t \cdot \frac{1}{2\left(\Delta_{2}+1\right)}\left|V_{1}\right| \geq\left|V_{1}\right| / 2$.

Since $\left(\alpha, \beta, \rho, f, \Delta_{1}, \Delta_{2}\right)$ is universal, the coloring restricted to the induced subgraph of $G$ with vertex set $V_{1}^{\prime}$ is $\left(\alpha, \beta, \rho, f, \Delta_{1}, \Delta_{2}\right)$-dense. Thus, there is $q^{\prime} \in\{1,2\}$, disjoint vertex subsets $T_{1}, \ldots, T_{u} \subset V_{1}^{\prime}$ each of cardinality at least $\alpha\left|V_{1}^{\prime}\right|$ and nonnegative integers $e_{1}, \ldots, e_{u}$ with $e_{1}+\cdots+e_{u}=\Delta_{q^{\prime}}-u+1$ such that the following holds:

- for $1 \leq i \leq u, T_{i}$ is $\operatorname{bi}-\left(f\left(\rho, e_{i}\right), \rho\right)$-dense in the graph of color $q^{\prime}$, and

- for $1 \leq i<j \leq u$, each vertex in $T_{i}$ is in at most $\beta\left|T_{j}\right|$ edges of color $3-q^{\prime}$ with vertices in $T_{j}$.

If $q^{\prime}=1$, we are done. Therefore, we may suppose $q^{\prime}=2$.

Note that $e_{1}+\cdots+e_{u}+d_{1}+\cdots+d_{t}=\Delta_{2}-u+1+\Delta_{2}-t+1=\left(2 \Delta_{2}+1\right)-(u+t)+1$. Moreover, $\beta \geq 4\left(\Delta_{2}+1\right) \rho$, implying that for all $1 \leq i \leq u$ and all $1 \leq j \leq t$ every vertex in $T_{i}$ is in at most $\beta\left|S_{j}\right|$ edges of color 1 with vertices in $S_{j}$. Therefore, the sequence $T_{1}, \ldots, T_{u}, S_{1}, \ldots, S_{t}$ implies that the edge-coloring of $G$ is $\left(\frac{1}{2} f\left(\rho, \Delta_{1}\right) \alpha, \beta, \rho, f, \Delta_{1}, 2 \Delta_{2}+1\right)$-dense, completing the proof.

By symmetry, the above lemma implies that if $\beta \geq 4\left(\Delta_{1}+1\right) \rho$ and $\left(\alpha, \beta, \rho, f, \Delta_{1}, \Delta_{2}\right)$ is universal, then $\left(\frac{1}{2} f\left(\rho, \Delta_{2}\right) \alpha, \beta, \rho, f, 2 \Delta_{1}+1, \Delta_{2}\right)$ is also universal.

Lemma 3.2 Let $h$ be a nonnegative integer and $f$ be such that $f(\rho, 0)=1$. Define

$$
\alpha_{h}=2^{-2 h} f(\rho, 0)^{-1} f\left(\rho, 2^{h}-1\right)^{-1} \prod_{i=0}^{h} f\left(\rho, 2^{i}-1\right)^{2} .
$$

Then $\left(\alpha_{h}, 2^{h+1} \rho, \rho, f, 2^{h}-1,2^{h}-1\right)$ is universal.

Proof: The proof is by induction on $h$. As already mentioned, if $\epsilon>1 / 2$, every graph $G$ is vacuously bi- $(\epsilon, \rho)$-dense. Since $\alpha_{0}=1>1 / 2$, setting $t=1$ and $S_{1}=V(G)$, we have $(1,2 \rho, \rho, f, 0,0)$ is universal, which is the base case $h=0$.

Suppose the lemma is satisfied for $h$, and we wish to show it for $h+1$. Let $D=2^{h}-1, D^{\prime}=2 D+1=$ $2^{h+1}-1$, and $\beta=4(D+1) \rho=2\left(D^{\prime}+1\right) \rho=2^{h+2} \rho$. Note that, for $\beta \geq \beta^{\prime}$, if $\left(\alpha, \beta^{\prime}, \rho, f, \Delta_{1}, \Delta_{2}\right)$ 
is universal then so is $\left(\alpha, \beta, \rho, f, \Delta_{1}, \Delta_{2}\right)$. Therefore, since $\left(\alpha_{h}, 2(D+1) \rho, \rho, f, D, D\right)$ is universal, $\left(\alpha_{h}, \beta, \rho, f, D, D\right)$ is also. Applying Lemma 3.1, we have that $\left(\frac{1}{2} f(\rho, D) \alpha_{h}, \beta, \rho, f, D, 2 D+1\right)$ is universal. Applying the symmetric version of Lemma 3.1 mentioned above, we have that

$$
\left(\frac{1}{2} f(\rho, 2 D+1) \frac{1}{2} f(\rho, D) \alpha_{h}, \beta, \rho, f, 2 D+1,2 D+1\right)=\left(\alpha_{h+1}, \beta, \rho, f, D^{\prime}, D^{\prime}\right),
$$

is universal, which completes the proof by induction.

A graph $G$ is $n$-Ramsey-universal if, in any 2-edge-coloring of $G$, there are monochromatic induced copies of every graph on $n$ vertices all of the same color. The following lemma implies Theorem 1.3.

Lemma 3.3 If $G$ is $(1 / 2, \lambda)$-pseudo-random on $N$ vertices with $\lambda \leq 2^{-140 n} n^{-40 n} N$, then $G$ is $n$ Ramsey-universal.

The set-up for the proof of this lemma is roughly similar to the one presented in the previous section. We start with a collection of bi-dense sets, in say blue, such that the density of red edges between each pair of sets is small. The goal is to embed a blue induced copy of a given graph $H$ on vertices $1, \ldots, n$. We embed vertices one at a time, always maintaining large sets in which we may embed later vertices. Suppose that at step $i$ of our embedding, after $v_{1}, v_{2}, \ldots, v_{i}$ are chosen, we have sets $V_{j, i}$ for $j>i$ corresponding to the possible choices for future $v_{j}$. If the vertices $j, \ell>i$ are not adjacent, then, by the pseudo-randomness of $G$, the density of nonedges between any two large sets is roughly $1 / 2$, and it is therefore easy to guarantee that we can pick $v_{j}$ and $v_{\ell}$ so that they are nonadjacent. On the other hand, if the vertices $j, \ell>i$ are adjacent, then we need to guarantee that $v_{j}$ and $v_{\ell}$ will be joined by a blue edge. Thus, it would be helpful to ensure that the density of blue edges between $V_{j, i}$ and $V_{\ell, i}$ is not too small. In the bounded-degree case we maintain such a property by exploiting the fact that the blue density between any two large sets is large. Here, we do not have this luxury in the case that $V_{j, i}$ and $V_{\ell, i}$ are subsets of different bi-dense sets in the collection. It is instead necessary to use the fact that the underlying graph $G$ is pseudo-random.

To see how this helps, suppose that we now wish to embed $v_{i+1}$. This will affect the sets $V_{j, i}$ and $V_{\ell, i}$, resulting in subsets $V_{j, i+1}$ and $V_{\ell, i+1}$. We would like these subsets to mirror the density properties between $V_{j, i}$ and $V_{\ell, i}$. The way we proceed is to show that using pseudo-randomness we can choose $v_{i+1}$ such that the density of red edges between the sets $V_{j, i+1}$ and $V_{\ell, i+1}$ remains small. Since $G$ is pseudo-random, the total density between large sets is roughly $1 / 2$ and therefore there will still be many blue edges between these two sets.

Proof of Lemma 3.3: We split the proof into four steps.

Step 1: We will first choose appropriate constants and prepare $G$ for embedding monochromatic induced subgraphs.

Any $(1 / 2, \lambda)$-pseudo-random graph on at least two vertices must satisfy $\lambda \geq 1 / 2$. Indeed, letting $A$ and $B$ be distinct vertex subsets each of cardinality 1 , we have

$$
1 / 2=|d(A, B)-1 / 2| \leq \frac{\lambda}{\sqrt{|A||B|}}=\lambda .
$$

It follows that $N \geq 2^{140 n} n^{40 n} \lambda \geq 2^{138 n} n^{40 n}$. 
We will start by picking some constants. Pick $\rho=2^{-13} n^{-3}, h=\lceil\log n\rceil \leq \log 2 n, \beta=2^{h+1} \rho \leq 8 n \rho=$ $2^{-10} n^{-2}, f(\rho, 0)=1$ and $f(\rho, d)=2^{-5 n} \rho^{d}$ if $d>0$, so

$$
\begin{aligned}
\alpha & =2^{-2 h} f(\rho, 0)^{-1} f\left(\rho, 2^{h}-1\right)^{-1} \prod_{i=0}^{h} f\left(\rho, 2^{i}-1\right)^{2}=2^{-2 h} f\left(\rho, 2^{h}-1\right) \prod_{i=1}^{h-1} f\left(\rho, 2^{i}-1\right)^{2} \\
& =2^{-2 h-5 n} \rho^{2^{h}-1} \prod_{i=1}^{h-1} 2^{-10 n} \rho^{2\left(2^{i}-1\right)}=2^{-2 h-(2 h-1) 5 n} \rho^{3 \cdot 2^{h}-2 h-3} \\
& \geq(2 n)^{-12 n} \rho^{6 n}=2^{-90 n} n^{-30 n} .
\end{aligned}
$$

Lemma 3.2 implies that $\left(\alpha, \beta, \rho, f, 2^{h}-1,2^{h}-1\right)$ is universal. As $2^{h} \geq n$, it follows that $(\alpha, \beta, \rho, f, n-$ $1, n-1)$ is also universal. Let $\epsilon_{1}=\frac{1}{2 n}, \epsilon_{2}=\frac{\epsilon_{1} \rho}{32 n}=2^{-19} n^{-5}, \epsilon_{3}=\epsilon_{4}=\frac{1}{8 n}, \epsilon_{5}=\frac{1}{8 n^{2}}, \epsilon_{6}=\epsilon_{2} \epsilon_{5}=2^{-22} n^{-7}$ and $\beta^{\prime}=2 n \beta \leq 2^{-9} n^{-1}$.

Since every red-blue edge of $G$ is $(\alpha, \beta, \rho, f, n-1, n-1)$-dense, we may assume that there are disjoint vertex subsets $S_{1}, \ldots, S_{t}$ each of cardinality at least $\alpha N$ and nonnegative integers $d_{1}, \ldots, d_{t}$ with $d_{1}+\cdots+d_{t}=n-t$ such that

- for $1 \leq i \leq t, S_{i}$ is bi- $\left(f\left(\rho, d_{i}\right), \rho\right)$-dense in the blue graph, and

- for $1 \leq i<j \leq t$, each vertex in $S_{i}$ is in at most $\beta\left|S_{j}\right|$ red edges with vertices in $S_{j}$.

We will show that we can find a monochromatic blue induced copy of each graph $H$ on $n$ vertices. We may suppose the vertex set of $H$ is $V(H)=[n]:=\{1, \ldots, n\}$. Partition $[n]=U_{1} \cup \ldots \cup U_{t}$, with the vertices in $U_{i}$ coming before the vertices in $U_{j}$ for $i<j$ and $\left|U_{i}\right|=d_{i}+1$ for $1 \leq i \leq t$. For $j \in U_{l}$, let $D(i, j)$ denote the number of neighbors $h$ of $j$ with $h \leq i$ and $h \in U_{l}$. Arbitrarily partition $S_{i}$ into $d_{i}+1$ sets $\bigcup_{k \in U_{i}} V_{k}$ each of cardinality at least $\left\lfloor\frac{\left|S_{i}\right|}{d_{i}+1}\right\rfloor \geq\left\lfloor\frac{\left|S_{i}\right|}{n}\right\rfloor \geq \frac{\left|S_{i}\right|}{2 n} \geq \frac{\alpha}{2 n} N$, where we use $d_{i}+1 \leq n$, $\left|S_{i}\right| \geq \alpha N$, and the lower bounds on $\alpha$ and $N$.

Step 2: We now describe our strategy for constructing induced blue copies of $H$. In broad outline, we proceed by induction, embedding each successive vertex $i$ in the set $V_{i}$. To achieve this, we have to maintain several conditions which allow us to embed future vertices.

At the end of step $i$, we will have vertices $v_{1}, \ldots, v_{i}$ and, for $j>i$, subsets $V_{j, i} \subset V_{j}$ such that the following four conditions hold.

1. for $j, \ell \leq i$, if $(j, \ell)$ is an edge of $H$, then $\left(v_{j}, v_{\ell}\right)$ is a blue edge of $G$, otherwise $v_{j}$ and $v_{\ell}$ are not adjacent in $G$;

2. for $j \leq i<\ell$, if $(j, \ell)$ is an edge of $H$, then $v_{j}$ is adjacent to all vertices in $V_{\ell, i}$ by blue edges, otherwise there are no edges of $G$ from $v_{j}$ to $V_{\ell, i}$;

3. for $j>i$, we have $\left|V_{j, i}\right| \geq 4^{-i} \rho^{D(i, j)}\left|V_{j}\right|$;

4. for $\ell>j>i$, if $j \in U_{q_{1}}$ and $\ell \in U_{q_{2}}$ with $q_{1}<q_{2}$, then each vertex in $V_{j, i}$ is in at most $\left(1+\epsilon_{1}\right)^{i} \beta^{\prime}\left|V_{\ell, i}\right|$ red edges with vertices in $V_{\ell, i}$.

Note that $V_{j, i}$ is a subset of vertices of $G$ in which we can still embed vertex $j$ from $H$ after $i$ steps of our embedding procedure. Clearly, at the end of the first $n$ steps of this process we obtain the required copy of $H$. For $i=0$ and $j \in[n]$, define $V_{j, 0}=V_{j}$. Notice that the above four properties are 
satisfied for $i=0$. Indeed, the first two properties are vacuously satisfied, the third property follows from $V_{j, 0}=V_{j}$, and the last property follows from the simple inequality $\beta^{\prime}\left|V_{\ell, 0}\right|=2 n \beta\left|V_{\ell}\right| \geq \beta\left|S_{q_{2}}\right|$. We now assume that the above four properties are satisfied at the end of step $i$ and show how to complete step $i+1$ by finding a vertex $v_{i+1} \in V_{i+1, i}$ and, for $j>i+1$, subsets $V_{j, i+1} \subset V_{j, i}$ such that conditions 1-4 still hold.

Before we begin the next step of the proof, we need to introduce some notation. For a vertex $w \in V_{j}$ and a subset $S \subset V_{\ell}$ with $j \neq \ell$, let

- $N(w, S)$ denote the set of vertices $s \in S$ such that $(s, w)$ is an edge of $G$,

- $R(w, S)$ denote the set of vertices $s \in S$ such that $(s, w)$ is a red edge of $G$,

- $B(w, S)$ denote the set of vertices $s \in S$ such that $(s, w)$ is a blue edge of $G$,

- $\tilde{N}(w, S)=N(w, S)$ if $(j, \ell)$ is an edge of $H$ and $\tilde{N}(w, S)=S \backslash N(w, S)$ otherwise,

- $\tilde{B}(w, S)=B(w, S)$ if $(j, \ell)$ is an edge of $H$ and $\tilde{B}(w, S):=S \backslash N(w, S)$ otherwise.

Note, for all $S \subset V_{\ell}$ and $w \in V_{j}$, that $\tilde{B}(w, S)=\tilde{N}(w, S) \backslash R(w, S)$. Moreover, since the graph $G$ is pseudo-random with edge density $1 / 2$, we expect that for every large subset $S \subset V_{\ell}$ and for most vertices $w \in V_{j}$ the size of $\tilde{N}(w, S)$ will be roughly $|S| / 2$.

Step 3: We next show that if there is a vertex satisfying certain conditions, then we can continue our embedding. In the last step we show that there is such a "good" vertex.

Let $q$ be the index such that $i+1 \in U_{q}$. Call a vertex $w \in V_{i+1, i}$ good if

1. for all $j>i+1$ such that $(j, i+1)$ is an edge of $H$ and $j \in U_{q},\left|B\left(w, V_{j, i}\right)\right| \geq \rho\left|V_{j, i}\right|$,

2. for all $j>i+1,\left|\tilde{N}\left(w, V_{j, i}\right)\right| \geq\left(\frac{1}{2}-\frac{\epsilon_{1}}{20}\right)\left|V_{j, i}\right|$,

3. for all $\ell>j>i+1$ with $j \in U_{q_{1}}, \ell \in U_{q_{2}}$, and $q_{1}<q_{2}$, there are at most $\epsilon_{2}\left|V_{j, i}\right|$ vertices $y \in V_{j, i}$ such that $y$ is in at least $\beta^{\prime}\left(\frac{1}{2}-\frac{\epsilon_{1}}{10}\right)\left|V_{\ell, i}\right|$ red edges with vertices in $V_{\ell, i}$ and $y$ is in at least $\left(\frac{1}{2}+\frac{\epsilon_{1}}{10}\right)\left|R\left(y, V_{\ell, i}\right)\right|$ red edges with vertices of $\tilde{N}\left(w, V_{\ell, i}\right)$.

Note that, because the graph $G$ is pseudo-random with edge density $1 / 2$, we expect a typical vertex in $V_{i+1, i}$ to be adjacent (and also nonadjacent) to roughly $1 / 2$ of the vertices in $V_{j, i}$ and $V_{\ell, i}$. Moreover, condition 3 roughly says that for a typical vertex, the density of red edges between its neighborhoods in $V_{j, i}$ and $V_{\ell, i}$ is not much larger than the overall density of red edges between these two sets.

We will now show that if there is a good vertex $w \in V_{i+1, i}$, then we may continue the embedding by taking $v_{i+1}=w$ and, for $j>i+1$ with $j \in U_{q_{1}}$, letting $V_{j, i+1}$ be the subset of $\tilde{B}\left(w, V_{j, i}\right)$ formed by deleting all vertices $y$ for which there is $\ell>j$ with $\ell \notin U_{q_{1}}$ such that $y$ is in at least $\beta^{\prime}\left(\frac{1}{2}-\frac{\epsilon_{1}}{10}\right)\left|V_{\ell, i}\right|$ red edges with vertices in $V_{\ell, i}$ and $y$ is in at least $\left(\frac{1}{2}+\frac{\epsilon_{1}}{10}\right)\left|R\left(y, V_{\ell, i}\right)\right|$ red edges with vertices of $\tilde{N}\left(w, V_{\ell, i}\right)$. Note that, by the third property of good vertices,

$$
\left|V_{j, i+1}\right| \geq\left|\tilde{B}\left(w, V_{j, i}\right)\right|-n \epsilon_{2}\left|V_{j, i}\right| .
$$

Let us verify each of the required properties of our embedding in turn.

To verify the first property, we need to show that if $j \leq i$ and $(j, i+1)$ is an edge of $H$ then $\left(v_{j}, v_{i+1}\right)$ is a blue edge and, if $(j, i+1)$ is not an edge of $H$, then $\left(v_{j}, v_{i+1}\right)$ is not in $G$. But this follows by 
induction since, when the first $i$ vertices were embedded, we had that for all $j \leq i<l$, if $(j, l)$ was an edge of $H$, then $v_{j}$ was adjacent to all edges of $V_{l, i}$ by blue edges. Otherwise, there were no edges between $v_{j}$ and $V_{l, i}$. Taking $l=i+1$, the necessary property follows.

For the second property, we would like to show that for $j \leq i+1<l$, if $(j, l)$ is an edge of $H$, then $v_{j}$ is adjacent to all vertices in $V_{l, i+1}$ by blue edges and, otherwise, there are no edges between $v_{j}$ and $V_{l, i+1}$. Observe that, for all $l>i+1$, the set $V_{l, i+1}$ is a subset of the set $V_{l, i}$. Therefore, by induction, we only need to check the condition for $j=i+1$. But $V_{l, i+1}$ is a subset of $\tilde{B}\left(v_{i+1}, V_{l, i}\right)$, so this follows by definition.

We now wish to prove that, for all $j>i+1,\left|V_{j, i+1}\right| \geq 4^{-(i+1)} \rho^{D(i+1, j)}\left|V_{j}\right|$. Inequality (1) together with the first property of good vertices implies that if $j>i+1,(j, i+1)$ is an edge of $H$ and $j \in U_{q}$ (recall that also $\left.i+1 \in U_{q}\right)$, then, since $\epsilon_{2} \leq \rho /(2 n)$ and $D(i+1, j)=D(i, j)+1$,

$$
\left|V_{j, i+1}\right| \geq\left(\rho-n \epsilon_{2}\right)\left|V_{j, i}\right| \geq \frac{\rho}{2}\left|V_{j, i}\right| \geq \frac{\rho}{2} 4^{-i} \rho^{D(i, j)}\left|V_{j}\right| \geq 4^{-(i+1)} \rho^{D(i+1, j)}\left|V_{j}\right| .
$$

Inequality (10), the second property of good vertices and the inductive assumption that $w$ has at most $\left(1+\epsilon_{1}\right)^{i} \beta^{\prime}\left|V_{j, i}\right|$ red neighbors in $V_{j, i}$ if $j \notin U_{q}$ together imply that for all other $j>i+1$, we have

$$
\begin{aligned}
\left|V_{j, i+1}\right| & \geq\left|\tilde{B}\left(w, V_{j, i}\right)\right|-n \epsilon_{2}\left|V_{j, i}\right|=\left|\tilde{N}\left(w, V_{j, i}\right) \backslash R\left(w, V_{j, i}\right)\right|-n \epsilon_{2}\left|V_{j, i}\right| \\
& \geq\left(\frac{1}{2}-\frac{\epsilon_{1}}{20}\right)\left|V_{j, i}\right|-\left(1+\epsilon_{1}\right)^{i} \beta^{\prime}\left|V_{j, i}\right|-n \epsilon_{2}\left|V_{j, i}\right| \geq\left(\frac{1}{2}-\frac{\epsilon_{1}}{20}-3 \beta^{\prime}-n \epsilon_{2}\right)\left|V_{j, i}\right| \\
& \geq\left(\frac{1}{2}-\frac{\epsilon_{1}}{10}\right)\left|V_{j, i}\right| \geq \frac{1}{4} 4^{-i} \rho^{D(i, j)}\left|V_{j}\right|=4^{-(i+1)} \rho^{D(i+1, j)}\left|V_{j}\right| .
\end{aligned}
$$

Here we use that $\epsilon_{1}=1 / 2 n, \beta^{\prime} \leq 2^{-9} n^{-1}, \epsilon_{2} \leq \epsilon_{1} / 32 n$, and $D(i+1, j)=D(i, j)$ (since $i+1$ and $j$ are either nonadjacent or belong to different $\left.U_{\mathrm{s}}\right)$. In either case, the required lower bound on the cardinality of $V_{j, i+1}$ holds. Note the intermediate inequality that $\left|V_{l, i+1}\right| \geq\left(\frac{1}{2}-\frac{\epsilon_{1}}{10}\right)\left|V_{l, i}\right|$ whenever $l \notin U_{q}$.

If $i+1<j<\ell$ is such that $j \in U_{q_{1}}$ and $\ell \in U_{q_{2}}$ with $q \leq q_{1}<q_{2}$, our deletion of vertices from $\tilde{B}\left(w, V_{j, i}\right)$ implies that each vertex in $V_{j, i+1}$ is in less than

$$
\beta^{\prime}\left(\frac{1}{2}-\frac{\epsilon_{1}}{10}\right)\left|V_{\ell, i}\right| \leq \beta^{\prime}\left|V_{\ell, i+1}\right|
$$

red edges with vertices in $V_{\ell, i}$ or each vertex in $V_{j, i+1}$ is in less than

$$
\begin{aligned}
\left(\frac{1}{2}+\frac{\epsilon_{1}}{10}\right)\left|R\left(y, V_{\ell, i}\right)\right| & \leq\left(\frac{1}{2}+\frac{\epsilon_{1}}{10}\right)\left(1+\epsilon_{1}\right)^{i} \beta^{\prime}\left|V_{\ell, i}\right| \leq\left(\frac{1}{2}+\frac{\epsilon_{1}}{10}\right)\left(1+\epsilon_{1}\right)^{i} \beta^{\prime}\left|V_{\ell, i+1}\right| /\left(\frac{1}{2}-\frac{\epsilon_{1}}{10}\right) \\
& \leq\left(1+\epsilon_{1}\right)^{i+1} \beta^{\prime}\left|V_{\ell, i+1}\right|
\end{aligned}
$$

red edges with vertices of $\tilde{N}\left(w, V_{\ell, i}\right)$. In either case, we see that the last desired condition of the embedding is satisfied.

Step 4: We have shown that if there is a good vertex, then we can continue the embedding. In this step we show that there is a good vertex in $V_{i+1, i}$, which completes the proof.

The next three claims imply that the fraction of vertices in $V_{i+1, i}$ that are good is at least $1-n \epsilon_{3}-$ $n \epsilon_{4}-n^{2} \epsilon_{5}>1 / 2$, i.e., more than half of the vertices of $V_{i+1, i}$ are good. Indeed, Claim 1 shows that the first property of good vertices is satisfied for all but at most $n \epsilon_{3}\left|V_{i+1, i}\right|$ vertices in $V_{i+1, i}$. Claim 2 
shows that the second property of good vertices is satisfied for all but at most $n \epsilon_{4}\left|V_{i+1, i}\right|$ vertices in $V_{i+1, i}$. Claim 3 shows that the third property of good vertices is satisfied for all but at most $n^{2} \epsilon_{5}\left|V_{i+1, i}\right|$ of the vertices in $V_{i+1, i}$. These three claims therefore complete the proof.

Claim 1 For $j>i+1$ such that $(j, i+1)$ is an edge of $H$ and $j \in U_{q}$, let $Q_{j}$ denote the set of vertices $w \in V_{i+1, i}$ such that $\left|B\left(w, V_{j, i}\right)\right|<\rho\left|V_{j, i}\right|$. Then $\left|Q_{j}\right|<\epsilon_{3}\left|V_{i+1, i}\right|$.

Proof: Suppose, for contradiction, that $\left|Q_{j}\right| \geq \epsilon_{3}\left|V_{i+1, i}\right|$. As $j, i+1 \in U_{q}$ and $\left|U_{q}\right|=d_{q}+1$, we have $d_{q} \geq 1$ and $f\left(\rho, d_{q}\right)=2^{-5 n} \rho^{d_{q}}$. Since $2^{3 n} \geq 8 n^{2},\left|V_{i+1, i}\right| \geq 4^{-i} \rho^{D(i, i+1)}\left|V_{i+1}\right|$ and $\left|V_{i+1}\right| \geq\left|S_{q}\right| / 2 n$, we have

$$
\left|Q_{j}\right| \geq \epsilon_{3} 4^{-i} \rho^{D(i, i+1)}\left|V_{i+1}\right| \geq \epsilon_{3} 4^{1-n} \rho^{d_{q}}\left|V_{i+1}\right| \geq \frac{\epsilon_{3}}{n} 4^{-n} \rho^{d_{q}}\left|S_{q}\right| \geq f\left(\rho, d_{q}\right)\left|S_{q}\right| .
$$

We also have

$$
\left|V_{j, i}\right| \geq 4^{-i} \rho^{D(i, j)}\left|V_{j}\right| \geq 4^{1-n} \rho^{d_{q}}\left|V_{j}\right| \geq n^{-1} 4^{-n} \rho^{d_{q}}\left|S_{q}\right| \geq f\left(\rho, d_{q}\right)\left|S_{q}\right| .
$$

Since $S_{q}$ is bi- $\left(f\left(\rho, d_{q}\right), \rho\right)$-dense in blue, the blue edge density between $Q_{j}$ and $V_{j, i}$ is at least $\rho$, contradicting the definition of $Q_{j}$.

Claim 2 For $j>i+1$, let $P_{j}$ denote the set of vertices $w \in V_{i+1, i}$ such that

$$
\left|\tilde{N}\left(w, V_{j, i}\right)\right|<\left(\frac{1}{2}-\frac{\epsilon_{1}}{20}\right)\left|V_{j, i}\right|
$$

Then $\left|P_{j}\right|<\epsilon_{4}\left|V_{i+1, i}\right|$.

Proof: The definition of $P_{j}$ implies that the density of edges between $P_{j}$ and $V_{j, i}$ is either less than $\frac{1}{2}-\frac{\epsilon_{1}}{20}$ or more than $\frac{1}{2}+\frac{\epsilon_{1}}{20}$ (depending on whether or not $(i+1, j)$ is an edge of $H$ ). Therefore, since $G$ is $(1 / 2, \lambda)$-pseudo-random, we have $\frac{\epsilon_{1}}{20}<\frac{\lambda}{\sqrt{\left|P_{j}\right|\left|V_{j, i}\right|}}$. Note that, for $j>i$, since $\rho=2^{-13} n^{-3}$ and $\alpha \geq 2^{-90 n} n^{-30 n}$,

$$
\left|V_{j, i}\right| \geq 4^{-i} \rho^{D(i, j)}\left|V_{j}\right| \geq 4^{-n} \rho^{n}\left|V_{j}\right| \geq 2^{-15 n} n^{-3 n} \frac{\alpha}{2 n} N \geq 2^{-106 n} n^{-34 n} N .
$$

Hence, since we also have $\epsilon_{1}=1 / 2 n, \epsilon_{4}=1 / 8 n$ and $\lambda \leq 2^{-140 n} n^{-40 n} N$,

$$
\begin{aligned}
\left|P_{j}\right| & <\frac{400 \lambda^{2}}{\epsilon_{1}^{2}\left|V_{j, i}\right|}<\frac{2^{9} \mid 2^{-280 n} n^{-80 n} N^{2}}{(2 n)^{-2} 2^{-106 n} n^{-34 n} N}=2^{11} n^{2} 2^{-174 n} n^{-46 n} N \\
& \leq 2^{-163 n} n^{-44 n} N \leq(8 n)^{-1} 2^{-106 n} n^{-34 n} N \leq \epsilon_{4}\left|V_{i+1, i}\right| .
\end{aligned}
$$

Claim 3 Fix a pair $j$ and $\ell$ with $i+1<j<\ell, j \in U_{q_{1}}$, $\ell \in U_{q_{2}}$, and $q_{1}<q_{2}$. Let $X=V_{i+1, i}$, $Y=V_{j, i}$, and $Z=V_{\ell, i}$. Define the bipartite graph $F=F_{j, \ell}$ with parts $X$ and $Y$ where $(x, y) \in X \times Y$ is an edge if

$$
|R(y, Z)| \geq \beta^{\prime}\left(\frac{1}{2}-\frac{\epsilon_{1}}{10}\right)|Z|
$$

and

$$
|R(y, Z) \cap \tilde{N}(x, Z)|>\left(\frac{1}{2}+\frac{\epsilon_{1}}{10}\right)|R(y, Z)| .
$$

Let $T_{j, \ell}$ denote the set of vertices in $X$ with degree at least $\epsilon_{2}|Y|$ in $F$. Then $\left|T_{j, \ell}\right| \leq \epsilon_{5}|X|$. 
Proof: For $y \in Y$, let $X_{y} \subset X$ denote the neighbors of $y$ in graph $F$. Note that, for every $x \in X_{y}$, the fact that $|R(y, Z) \cap \tilde{N}(x, Z)|>\left(\frac{1}{2}+\frac{\epsilon_{1}}{10}\right)|R(y, Z)|$ implies that, in either the graph $G$ or its complement, $x$ has at least $\left(\frac{1}{2}+\frac{\epsilon_{1}}{10}\right)|R(y, Z)|$ neighbors in $R(y, Z)$ (this is again because $\tilde{N}(x, Z)$ is either the neighborhood of $x$ or its complement depending on whether or not $(i+1, \ell)$ is an edge of $H)$. Therefore, since $G$ is $(1 / 2, \lambda)$-pseudo-random,

$$
\frac{\epsilon_{1}}{10} \leq \frac{\lambda}{\sqrt{\left|X_{y}\right||R(y, Z)|}}
$$

Note that, by the first condition on $F$, if $y$ has any neighbors in $X,|R(y, Z)| \geq \beta^{\prime}|Z| / 4$. Therefore,

$$
\left|X_{y}\right| \leq \frac{100 \lambda^{2}}{\epsilon_{1}^{2}|R(y, Z)|} \leq \frac{400 \lambda^{2}}{\epsilon_{1}^{2} \beta^{\prime}|Z|} \leq \epsilon_{6}|X|
$$

This last inequality follows as in the previous claim. Indeed, since $\beta^{\prime}=2^{-9} n^{-1}, \epsilon_{6}=2^{-22} n^{-7}$, $Z=V_{\ell, i}$, and $X=V_{i+1, i}$, using inequalities (213), we have

$$
\frac{400 \lambda^{2}}{\epsilon_{1}^{2} \beta^{\prime}|Z|} \leq \beta^{\prime-1} 2^{-163 n} n^{-44 n} N \leq 2^{-154 n} n^{-43 n} N \leq 2^{-22} n^{-7} 2^{-106 n} n^{-34 n} N \leq \epsilon_{6}|X| .
$$

Therefore, the edge density of $F$ between $X$ and $Y$ is at most $\epsilon_{6}$ and there are at most $\frac{\epsilon_{6}|X||Y|}{\epsilon_{2}|Y|}=\epsilon_{5}|X|$ vertices in $X$ with degree at least $\epsilon_{2}|Y|$ in $F$.

\section{Concluding remarks}

Another interesting concept of sparseness, introduced by Chen and Schelp [3], is that of arrangeability. A graph $H$ is said to be $p$-arrangeable if there is an ordering of the vertices of $H$ such that, for any vertex $v_{i}$, the set of neighbors to the right of $v_{i}$ in the ordering have at most $p$ neighbors to the left of $v_{i}$ (including $v_{i}$ itself). Extending the result of Chvátal, Rödl, Szemerédi and Trotter [4], Chen and Schelp showed that for every $p$ there is a constant $c(p)$ such that, for any $p$-arrangeable graph $H$ with $n$ vertices, $r(H) \leq c(p) n$. This result has several consequences. Planar graphs, for example, may be shown to be 10-arrangeable [24, so their Ramsey numbers grow linearly. The best bound that is known for $c(p)$, again due to Graham, Rödl and Ruciński [22], is $c(p) \leq 2^{c p(\log p)^{2}}$. Unfortunately, it is unclear whether the bounds that we have given for bounded-degree graphs can be extended to the class of arrangeable graphs. It would be interesting to prove such a bound.

An even more problematic notion is that of degeneracy. A graph $H$ is said to be $d$-degenerate if there is an ordering of the vertices of $H$ such that any vertex $v_{i}$ has at most $d$ neighbors that precede it in the ordering. Equivalently, every subgraph of $H$ has a vertex of degree at most $d$. A conjecture of Burr and Erdős [2] states that for every $d$ there should be a constant $c(d)$ such that, for any $d$-degenerate graph $H$ with $n$ vertices, $r(H) \leq c(d) n$. This conjecture, which is still open, is a substantial generalization of the results on Ramsey numbers of bounded-degree graphs. The best result that is known, due to Fox and Sudakov [20], is $r(H) \leq 2^{c(d) \sqrt{\log n}} n$.

An old related problem is to bound the Ramsey number of graphs with $m$ edges. Erdös and Graham 15] conjectured that among all graphs with $m=\left(\begin{array}{l}n \\ 2\end{array}\right)$ edges and no isolated vertices, the complete graph on $n$ vertices has the largest Ramsey number. Motivated by the lack of progress on this conjecture, Erdős [12] asked whether one could at least show that the Ramsey number of any graph with $m$ edges 
is not much larger than that of the complete graph with the same size. Since the number of vertices in a complete graph with $m$ edges is on the order of $\sqrt{m}$, Erdös conjectured that $r(H) \leq 2^{c \sqrt{m}}$ holds for every graph $H$ with $m$ edges and no isolated vertices. Until recently the best known bound for this problem was $2^{c \sqrt{m} \log m}$ (see [1]). To attack Erdös' conjecture one can try to use the result on Ramsey numbers of bounded-degree graphs. Indeed, given a graph $H$ with $m$ edges, one can first embed the $2 \sqrt{m}$ vertices of largest degree in $H$ using the standard pigeonhole argument of Erdös and Szekeres [17]. The remaining vertices of $H$ span a graph with maximum degree $\sqrt{m}$. Hence, one may apply the arguments used to prove the upper bound for Ramsey numbers of bounded-degree graphs to embed the rest of $H$. However, this approach will likely require an upper bound of $2^{c \Delta} n$ on the Ramsey number for graphs on $n$ vertices of maximum degree $\Delta$, which we do not have yet. Recently, the third author [33] was able to circumvent this difficulty and prove Erdös' conjecture.

Finally, we would like to stress that the proofs given in this paper are highly specific to the 2-color case. The best results that are known in the $q$-color case are obtained by an entirely different method [19] and are considerably worse. For example, the $q$-color Ramsey number $r_{q}(H)$ of a graph on $n$ vertices with maximum degree $\Delta$ is only known to satisfy the inequality $r_{q}(H) \leq 2^{c_{q} \Delta^{2}} n$. It would be of considerable interest to improve this latter bound to $r_{q}(H) \leq 2^{c_{q} \Delta^{1+o(1)}} n$.

\section{References}

[1] N. Alon, M. Krivelevich, B. Sudakov, Turán numbers of bipartite graphs and related Ramsey-type questions, Combin. Probab. Comput. 12 (2003), 477-494.

[2] S.A. Burr and P. Erdős, On the magnitude of generalized Ramsey numbers for graphs, in Infinite and Finite Sets, Vol. 1 (Keszthely, 1973), Colloq. Math. Soc. János Bolyai, Vol. 10, North-Holland, Amsterdam/London, 1975, 215-240.

[3] G. Chen and R. Schelp, Graphs with linearly bounded Ramsey numbers, J. Combin. Theory Ser. B 57 (1993), 138-149.

[4] V. Chvatál, V. Rödl, E. Szemerédi and W.T. Trotter Jr., The Ramsey number of a graph with bounded maximum degree, J. Combin. Theory Ser. B 34 (1983), 239-243.

[5] D. Conlon, A new upper bound for diagonal Ramsey numbers, Annals of Math. 170 (2009), 941960.

[6] D. Conlon, Hypergraph packing and sparse bipartite Ramsey numbers, Combin. Probab. Comput., 18 (2009), 913-923.

[7] D. Conlon, J. Fox and B. Sudakov, Ramsey numbers of sparse hypergraphs, Random Structures Algorithms 35 (2009), 1-14.

[8] O. Cooley, N. Fountoulakis, D. Kühn and D. Osthus, 3-uniform hypergraphs of bounded degree have linear Ramsey numbers, J. Combin. Theory Ser. B 98 (2008), 484-505.

[9] O. Cooley, N. Fountoulakis, D. Kühn and D. Osthus, Embeddings and Ramsey numbers of sparse k-uniform hypergraphs, Combinatorica 28 (2009), 263-297. 
[10] W. Deuber, A generalization of Ramsey's theorem, in Infinite and Finite Sets, Vol. 1 (Keszthely, 1973), Colloq. Math. Soc. János Bolyai, Vol. 10, North-Holland, Amsterdam/London, 1975, 323332.

[11] N. Eaton, Ramsey numbers for sparse graphs, Discrete Math. 185 (1998), 63-75.

[12] P. Erdős, On some problems in graph theory, combinatorial analysis and combinatorial number theory, in Graph theory and combinatorics (Cambridge, 1983), Academic Press, London, New York, 1984, 1-17.

[13] P. Erdős, Problems and results on finite and infinite graphs, in Recent advances in graph theory (Proc. Second Czechoslovak Sympos., Prague, 1974), Academia, Prague, 1975, 183-192.

[14] P. Erdős, Some remarks on the theory of graphs, Bull. Amer. Math. Soc. 53 (1947), 292-294.

[15] P. Erdőos and R. Graham, On partition theorems for finite graphs, in Infinite and finite sets (Colloq., Keszthely, 1973), Vol. I; Colloq. Math. Soc. János Bolyai, Vol. 10, North-Holland, Amsterdam, $1975,515-527$.

[16] P. Erdős, A. Hajnal and L. Pósa, Strong embeddings of graphs into colored graphs, in Infinite and Finite Sets, Vol. 1 (Keszthely, 1973), Colloq. Math. Soc. János Bolyai, Vol. 10, North-Holland, Amsterdam/London, 1975, 585-595.

[17] P. Erdős and G. Szekeres, A combinatorial problem in geometry, Compositio Math. 2 (1935), 463-470.

[18] J. Fox and B. Sudakov, Induced Ramsey-type theorems, Adv. Math. 219 (2008), 1771-1800.

[19] J. Fox and B. Sudakov, Density theorems for bipartite graphs and related Ramsey-type results, Combinatorica 29 (2009), 153-196.

[20] J. Fox and B. Sudakov, Two remarks on the Burr-Erdős conjecture, European J. Combinatorics, 30 (2009), 1630-1645.

[21] W.T. Gowers, Lower bounds of tower type for Szemerédi's uniformity lemma, Geom. Funct. Anal. 7 (1997), 322-337.

[22] R.L. Graham, V. Rödl and A. Ruciński, On graphs with linear Ramsey numbers, J. Graph Theory 35 (2000), 176-192.

[23] R.L. Graham, V. Rödl and A. Ruciński, On bipartite graphs with linear Ramsey numbers, Combinatorica 21 (2001), 199-209.

[24] H.A. Kierstead and W.T. Trotter Jr., Planar graph colorings with an uncooperative partner, J. Graph Theory 18 (1994), 569-584.

[25] Y. Kohayakawa, H. Prömel, and V. Rödl, Induced Ramsey numbers, Combinatorica 18 (1998), 373-404.

[26] A. V. Kostochka and B. Sudakov, On Ramsey numbers of sparse graphs, Combin. Probab. Comput. 12 (2003), 627-641. 
[27] M. Krivelevich and B. Sudakov, Pseudorandom graphs, in More Sets, Graphs and Numbers, Bolyai Society Mathematical Studies 15, Springer, 2006, 199-262.

[28] L. Lovász, On decomposition of graphs, Studia Sci. Math. Hungar. 1 (1966), 237-238.

[29] B. Nagle, S. Olsen, V. Rödl and M. Schacht, On the Ramsey number of sparse 3-graphs, Graphs and Combinatorics 27 (2008), 205-228.

[30] F.P. Ramsey, On a problem of formal logic, Proc. London Math. Soc. Ser. 230 (1930), 264-286.

[31] V. Rödl, The dimension of a graph and generalized Ramsey theorems, Master's thesis, Charles University, 1973.

[32] J. Spencer, Ramsey's theorem - a new lower bound, J. Comb. Theory Ser. A 18 (1975), 108-115.

[33] B. Sudakov, A conjecture of Erdős on graph Ramsey numbers, submitted.

[34] E. Szemerédi, Regular partitions of graphs, in Problémes Combinatoires et Théorie des Graphes (Orsay 1976), Colloq. Internat. CNRS, 260, CNRS, Paris, 1978, 399-401.

[35] A. Thomason, Pseudorandom graphs, in Random graphs '85 (Poznań, 1985), North-Holland Math. Stud., Vol. 144, North-Holland, Amsterdam, 1987, 307-331.

[36] A. Thomason, Random graphs, strongly regular graphs and pseudorandom graphs, in Surveys in Combinatorics 1987, London Math. Soc. Lecture Note Ser., Vol. 123, Cambridge Univ. Press, Cambridge, 1987, 173-195. 